\title{
Plumbagin inhibits proliferation and induces apoptosis of hepatocellular carcinoma by downregulating the expression of SIVA
}

This article was published in the following Dove Press journal:

Drug Design, Development and Therapy

\section{Tingting $\mathrm{Li}$ \\ Mengjiao Lv \\ Xiaohua Chen \\ Yongsheng Yu \\ Guoqing Zang \\ Zhenghao Tang}

Department of Infectious Disease, Shanghai Jiao Tong University Affiliated Sixth People's Hospital, Shanghai, 200233, People's Republic of China
Correspondence: Zhenghao Tang:

Guoqing Zang

Shanghai Jiao Tong University Affiliated

Sixth People's Hospital, 600 Yishan Road,

Shanghai 200233, People's Republic of

China

$\mathrm{Tel}+8602124058673$

Fax +8602124058673

Email tzhhao@I63.com;

zangguoqin@।26.com
Purpose: Plumbagin is thought to be a bioactive phytochemical drug and exerts an antitumor effect on various cancers. However, few studies focus on the antitumor activity of plumbagin on liver cancer. This study first investigated the antitumor activity of plumbagin on liver cancer and further investigated the molecular mechanism of its antitumor activity against hepatocellular carcinoma, both in vitro and in vivo.

Methods: The antiproliferative activity of plumbagin was evaluated through CCK-8, EdU, and colony forming test. The cell cycle and apoptosis were then analyzed by flow cytometer. Western blot was used to detect the expression of apoptosis related protein, SIVA, and mTOR pathway. RNA-seq was performed to determine the gene expression profiles and overexpressed or knocked down SIVA to validate its role in plumbagin's antitumor activity. Regarding animal experiment, a xenograft model in BALB/c nude mice was built using LM3-Luci cells. Then bioluminescence imaging and further immunohistochemistry were performed to study the antitumor activity and the expression of SIVA and mTOR in the plumbagin-treated group.

Results: Plumbagin can inhibit proliferation and induce apoptosis of liver cancer cells in vitro. Further experiment demonstrated that plumbagin could inhibit the expression of SIVA and subsequently downregulate the mTOR signaling pathway, and upregulating the expression of SIVA will alleviate the antitumor activity of plumbagin on liver cancer, which confirmed the important role of the SIVA/mTOR signaling pathway in the antitumor activity of plumbagin. In vivo bioluminescence imaging showed a decreased signal in the plumbagintreated group, and further immunohistochemistry demonstrated that plumbagin could inhibit the SIVA/mTOR signaling pathway in tumor tissues.

Conclusion: Our promising results showed that plumbagin could inhibit proliferation and induce apoptosis of hepatic cancer through inhibiting the SIVA/mTOR signaling pathway for the first time, which indicated that plumbagin might be a good candidate against liver cancer.

Keywords: hepatocellular carcinoma, plumbagin, antitumor, apoptosis, SIVA, mTOR

\section{Introduction}

Hepatocellular carcinoma (HCC) is the most common cancer in liver. ${ }^{1}$ It is reported that $\mathrm{HCC}$ has become a global health problem and more than a million of people were diagnosed with HCC per year worldwide, ${ }^{2}$ which ranks fourth in all diagnosed cancers. ${ }^{1}$ Regarding China, HCC is the fourth cause of cancer deaths, which may be due to the high morbidity of chronic hepatitis $\mathrm{B}$ and $\mathrm{C}$ virus infections in China. ${ }^{3}$ The tumorigenesis and progression of HCC will 
definitely impair the metabolism and detoxification function of liver, and $\mathrm{HCC}$ is a malignancy which can metastasize to other organs and finally result in death. ${ }^{4}$ To date, the treatment of HCC is various and individual. Surgical resection, liver transplantation, chemotherapy, radiotherapy, and transcatheter arterial chemoembolization (TACE) are all employed to treat HCC clinically according to the conditions of diseases. ${ }^{5-7}$ However, most of the patients diagnosed with HCC were at an advanced stage and in liver dysfunction status. ${ }^{8}$ The high rate of recurrence and poor tolerance with chemotherapy or radiotherapy means only $15 \%$ of HCC patients can obtain curative treatments. ${ }^{9}$ Therefore, there is an urgent issue to find an effective, tolerable, and safe treatment to prevent and treat HCC. Recently, some studies have revealed that the extracts of some natural plants or traditional medicine exerted antitumor efficacy against HCC, which indicates the promising application of natural components. ${ }^{10,11}$

Plumbagin, a plant-derived quinoid constituent, is first isolated from the roots of the medicinal herb plumbago and exerts diverse pharmacological effects. ${ }^{12}$ Specifically, plumbagin has been used in treating allergic reaction, infections, and some other ailments for more than 2000 years in China and India. ${ }^{13}$ In recent years, some studies have demonstrated that plumbagin could inhibit cell proliferation, tumor metastasis, and induce apoptosis of various kinds of cancers. ${ }^{14,15}$ To be specific, plumbagin can inhibit intestinal tumors in rats when fed in their diet with a concentration of $200 \mathrm{ppm} .{ }^{16}$ Plumbagin also exerts antitumor activity against prostate cancer, lung cancer, esophageal cancer, etc. ${ }^{14,15,17}$ Plumbagin was employed to overcome drug resistance of pancreatic cancer. ${ }^{18}$ However, few studies focus on the anticancer activity of plumbagin on HCC, and the anticancer efficacy and mechanism of plumbagin remain unclear. Some studies reported that the suppression of Ras signaling, STAT3-PLK1-AKT pathway, and AKT/ mTOR pathway was responsible for the cytotoxicity of plumbagin. ${ }^{15,17,18}$ Recently, SIVA, an apoptosis regulator protein that can bind to CD27 and regulate cell death, was reported to play a crucial role in activating mTOR signaling and contribute to tumorigenesis. ${ }^{19}$ Inhibiting the expression of SIVA can induce apoptosis and autophagy of lung cancer cells. ${ }^{20}$ In this study, we first evaluated the antiproliferation and apoptosis inducing activity of plumbagin on HCC cell lines (HepG2 and LM3) in vitro, and further investigated the potential mechanism of plumbagin's antitumor ability. Then an in vivo xenograft model was built to confirm the antitumor activity of plumbagin and its mechanism in vivo. Our results indicate that plumbagin can effectively inhibit proliferation and induces apoptosis of hepatocellular carcinoma, which is, in part, due to the inhibition of the SIVA/mTOR signaling pathway.

\section{Methods and materials \\ Cell culture}

Human hepatocellular carcinoma cell lines (HepG2 and LM3) were purchased from the Chinese Academy of Science cell bank and cultured in DMEM (HyClone, USA) supplemented with $10 \%$ fetal bovine serum (Gibco, USA) and $1 \%$ penicillin/ streptomycin (Gibco) at $37^{\circ} \mathrm{C}$ in a humidified atmosphere of $5 \% \mathrm{CO}_{2}$. Cells were passaged by trypsinization when reaching $90 \%$ confluence. LM3-Luci was constructed by stable expression of luciferase in LM3 by lentivirus vector (Genechem, China). Plumbagin and MHY1485 (a cell-permeable activator of mTOR) were purchased from Sigma-Aldrich. The purity of plumbagin is $\geq 98 \%$ and its molecular formula is $\mathrm{C}_{11} \mathrm{H}_{8} \mathrm{O}_{3}$. Cells were treated with drugs at a particular concentration and for particular times. Control cells treated with equivalent DMSO were also included.

\section{Cell viability}

HepG2 and LM3 were seeded at 5,000 cells per well in 96well and treated with a series of PL concentration for 24, 48, and 72 hours. Then cells were rinsed with PBS and incubated in 10\% CCK-8-containing medium for 4 hours at $37^{\circ} \mathrm{C}$, and the absorbance of $450 \mathrm{~nm}$ was acquired by a spectrophotometer (Bio-Rad, USA).

The cell proliferation was evaluated by Edu incorporation assay. Cells (HepG2 and LM3) were seeded in a 24well plate at a density of $2 \times 10^{5} / \mathrm{ml}$ and treated with 2.5 $\mu \mathrm{M}, 5 \mu \mathrm{M}$ plumbagin containing medium for 48 hours. Then cells were stained with Edu kit (Beyotime, China) according to the manufacturer's instruction. Images were obtained by fluorescence microscopy (Leica, Germany).

A colony forming experiment was performed by seeding 500 cells per well in a 6-well plate and treating with $2.5 \mu \mathrm{M}, 5 \mu \mathrm{M}$ plumbagin containing medium for 10 days. Then cells were washed with PBS, fixed with $4 \%$ paraformaldehyde (PFA) for 30 minutes and stained with $1 \%$ crystal violet for 15 minutes. Images were photographed by a digital scanner. 


\section{Cell apoptosis assay}

Cells were cultured with the indicated treatment containing medium for 24 hours. Then cells were collected and stained with AnnexinV-FITC and PI doubled stained apoptosis kit (Donjindo, Shanghai) according to the manufacturer's instruction. The signals were detected by flow cytometer (Beckham Coulter, USA). AnnexinV-FITC positive cells were defined as apoptotic cells.

\section{Cell cycle analysis}

Cells were collected and fixed with $4 \%$ PFA overnight at $4{ }^{\circ} \mathrm{C}$ after being treated with indicated plumbagin for 24 hours. The cell cycle distribution was analyzed according to the cell cycle analysis kit (Beyotime) and detected with a flow cytometer (Beckham Coulter).

\section{Transcriptome analysis}

We employed a RNA sequencing technique to analyze the gene expression profile of LM3 cells when treated with 5 $\mu \mathrm{M}$ plumbagin for $24 \mathrm{~h}$. Then, cell RNA was harvested by TRIzol reagent, and the whole gene expression was examined at the Beijing Genomics Institute (BGI, China). Fold changes in the expression of selected genes were exhibited by a heat map, and pathway enrichment was evaluated by KEGG pathway analysis.

\section{Knowdown and overexpression of SIVA}

A pCMV6-Entry vector system containing the open reading frame of the SIVA gene was used to overexpress SIVA in LM3 and HepG2 according to the manufacturer's instruction (SIVA (NM_006427) Human Tagged ORF Clone, Origene, USA). An empty plasmid vector was used as a negative control (Origene). Plasmids were transfected mediated by Lipofectamine 3000 for 48 hours before drug treatments.

For the SIVA knockdown experiment, RNAi silencing was performed with three unique 27-mer siRNA duplexes targeting SIVA (human) transcripts (Origene). The trilencer-27 Universal Scrambled siRNA Duplex was used as a negative Control (Origene). Transient transfection was carried out in Opti-MEM mediated by Lipofectamine RNAiMAX according to the manufacturer's recommendations (Thermo Fisher Scientific, USA). After 48-72 hours, cells were collected for further analyses.

\section{Western blotting}

Cells were harvested and lysed with RIPA buffer (1\% NP40, $0.5 \%$ sodium deoxycholate, $0.1 \%$ SDS, Tris $\mathrm{pH} 8.0$ ). Protease inhibitors (Beyotime) were also added. Then 30 $\mu \mathrm{g}$ cell lysates were loaded and separated using sodium dodecyl sulfate-polyacrylamide gel electrophoresis (SDSPAGE, 10\%) and subsequently blotted on polyvinylidene fluoride (PVDF) membrane. After blocking with 5\% nonfat milk for 1 hour, the membrane was incubated overnight at $4^{\circ} \mathrm{C}$ with the following primary antibodies: caspase 3 and cleaved caspase3 (1:1,000 dilution, CST, USA); cleaved PARP (1:1,000 dilution, CST); SIVA (1:500 dilution, CST); p53 (1:1,000 dilution, CST); mTOR (1:1,000 dilution, CST); phospho-mTOR (1:500 dilution, CST, USA); S6K (1:1000 dilution, CST, USA); phospho-S6K (1:500 dilution, CST, USA); 4EBP1 (1:1000 dilution, CST, USA) phospho4EBP1 (1:1500 dilution, CST, USA) ; GAPDH (1:1000 dilution, CST, USA). Then proper HRP-conjugated IgG secondary antibodies (Jackson ImmunoResearch, USA) were used to mark primary antibodies . Finally, the immunoreactive bands were visualized by immobilon Western chemiluminescent HRP substrates (Merck, Millipore, USA).

\section{Murine ectopic HCC allograft model}

The animal experiments were approved by the Animal Care Committee of Shanghai Jiao Tong University Affiliated Sixth People's Hospital, and all the animal welfare and experimental procedures were in accordance with the National Institutes of Health Guide for Care and Use of Laboratory Animals (GB14925-2010). Forty 6-8 weeks BALB/c-nu/nu mice (Slac, China) were employed in this study. Mice ( $\mathrm{n}=5 /$ cage) were housed under pathogen-free conditions of a 12 hour dark-light cycle for 14 days prior to experiments. LM3-luciferase cells $\left(1 \times 10^{7}\right)$ were suspended in $100 \mu \mathrm{LMEM}$ medium and injected subcutaneously into the flank of mice. At $100 \mathrm{~mm}^{3}$ tumor volume, animals were randomly divided into two groups. The control group was treated with vehicle alone, while the PL group were treated with plumbagin $2.5 \mathrm{mg} / \mathrm{kg} /$ day intraperitoneally five times a week. Tumor size was calculated as: volume $\left(\mathrm{mm}^{3}\right)$ $=0.5 \times(\text { width })^{2} \times$ length. Tumor volume was monitored once per week using a digital hand-caliper. Body weight was also measured weekly to assess potential sideeffects due to the administration of the drug. After 4 weeks treatment, the bioluminescent signals of tumors were obtained by an IVIS Lumina XRMS Series III 
imaging system. The mouse was anesthetized through inhalation of isoflurane (2\%) during the examination, and the photon signals were obtained and recorded as pseudo-color images. The average intensity of the signals was calculated to indicate bacterial burden through IVIS software (Caliper Life Sciences, Perkin-Elmer). Then animals were sacrificed with an overdose of pentobarbital. Tumors were harvested, weighted, recorded, and fixed for further analysis.

\section{Immunohistochemistry staining}

To determine the expression of SIVA and p-mTOR in tumor tissues, we performed immunohistochemistry staining on $4 \mu \mathrm{m}$ thick paraffin embedded sections. Briefly, sections were incubated with primary antibody at $4^{\circ} \mathrm{C}$ for 12 hours. Then, secondary antibody was applied to attach to the primary antibody for 2 hours. As to immunohistochemistry, a color reaction was developed with diaminobenzidine. Then representative images were obtained using optical microscopy .

\section{Statistical analysis}

SPSS 22.0 program (SPSS Inc., Chicago, IL, USA) was applied to perform statistical analysis. Data were represented as mean $\pm \mathrm{SD}$. Experimental results were statistically analyzed by the Student's independent $t$-test or one-way ANOVA. Log-rank (Mantel-Cox) tests were employed to compare the differences of survival curves. A $P$-value $<0.05$ was considered statistically significant.

\section{Results}

\section{Plumbagin inhibits the proliferation of $\mathrm{HCC}$}

To assess the potential treatment of PL for $\mathrm{HCC}$, we first examined the cytotoxicity of PL against hepatocellular carcinoma cell lines (HepG2 and LM3). As depicted in Figure 1A, PL effected the HCC cell lines in a time-dependent and dose-dependent way, which the viability of HepG2 and LM3 was undermined along with the increasing PL concentration and treatment time. The half maximal inhibitory concentration (IC50) for HepG2 was higher than that of LM3, as shown in Figure 1A. Next, we observed the proliferative capacity of cells upon PL treatment by assessing EdU incorporation and colony formation. Perturbation with different PL concentrations resulted in a significant decrease in the percentage of EdU- positive cells in LM3, while the percentage of EdUpositive cells in HepG2 was significantly decreased only with higher PL concentrations (Figure 1B). Similarly, PL markedly inhibited the colony forming capacity in the low-density plating assay of LM3 and HepG2 in a dose-dependent way (Figure 1C).

\section{Plumbagin induces cell cycle arrest and apoptosis of HCC}

To decipher the underlying mechanisms attributed to the PL-mediated inhibition of cell proliferation, we performed the cell cycle and apoptosis analysis. Cell cycle distribution performed by flow cytometry in the G2/M phase was revealed to be augmented as PL concentration enhanced (Figure 2), indicating that PL could induce a G2/M-phase arrest in HCC cell lines. In addition, PL could induce apoptosis of LM3 and HepG2 in a dose-dependent way (Figure 3A). Western blot analysis showed that the proapoptotic markers cleaved caspase 3 and cleaved PARP could be activated by PL in both cell lines (Figure 3B), suggesting the caspase pathway facilitated PL-induction apoptosis of HCC cell lines.

\section{Plumbagin suppressed the mTOR pathway by reducing the expression of SIVA}

To determine the molecular mechanism conferring to PL cytotoxicity, we determine the gene expression profile of plumbagin-treated cells (LM3) using RNAseq. Our transcriptome analysis results showed a total of 494 genes differentially expressed between before and after drug treatment in LM3 ( $>1.5$-fold, $p<0.05)$. The heat map of the representative genes was shown in Figure 4A, depicting the expression fold changes between the control group and plumbagin-treated group. To be specific, the expression levels of apoptosis-associated genes such as caspase 3 and PARP were elevated in the plumbagin-treated group (Figure 4A), and the $\log _{2}$ (fold-change) were 1.11 and 1.45 for caspase 3 and PARP, respectively. In addition, the expression of SIVA and mTOR, two hyperactivated genes in cancer, were downregulated in the plumbagin-treated group (Figure 4A), and the $\log _{2}$ (fold-change) were -2.33 and -0.84 for SIVA and $\mathrm{mTOR}$, respectively. Moreover, kyoto encyclopedia of genes and genomes (KEGG) pathway analysis was utilized to show the upregulated and downregulated pathways (Figures 4B 
A

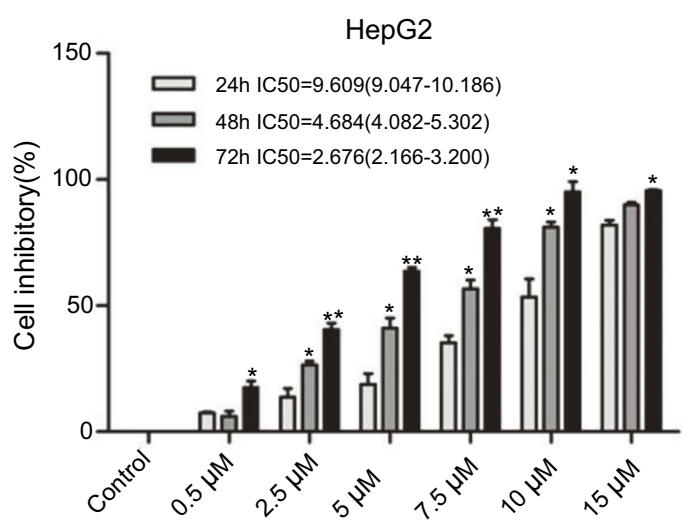

LM3

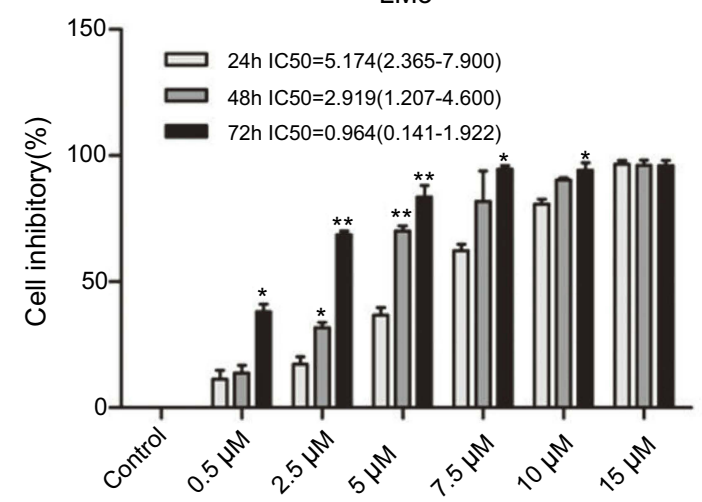

B
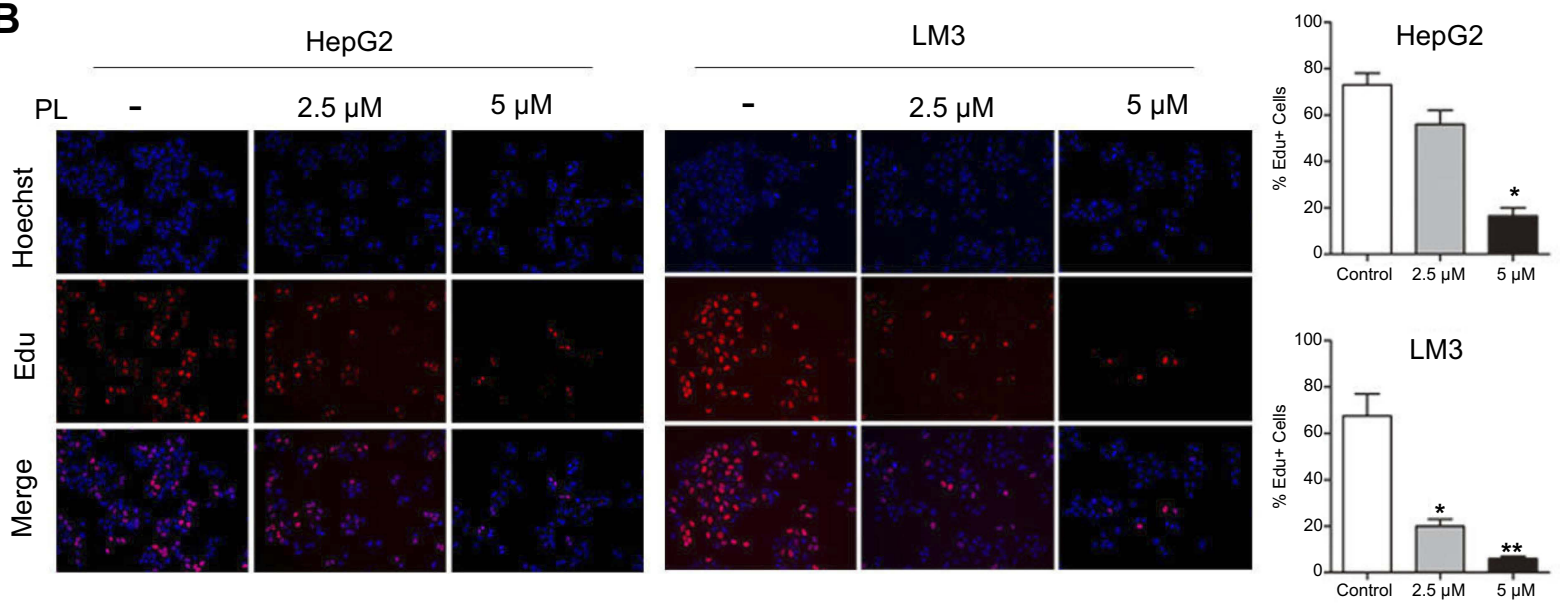

C

HepG2

LM3
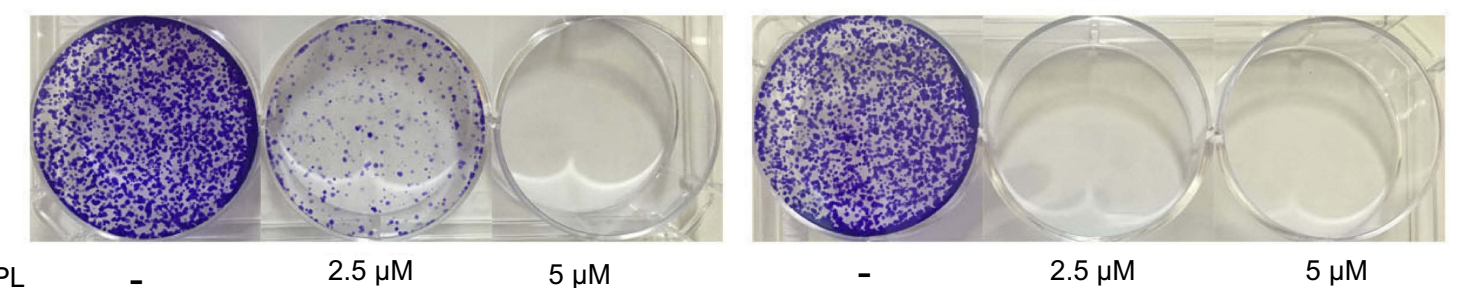

Figure I Anti-proliferative effect of plumbagin on HCC cell lines. (A) Cell inhibitory rate of plumbagin against HepG 2 and LM3 cells determined by CCK-8 test, *P<0.05, $* * P<0.01$ vs 24 hour group. (B) The results of EdU fluorescent staining, $* P<0.05$, $* * P<0.0$ I vs control group. (C) The inhibitory effect of plumbagin on the colony forming capacity of HepG2 and LM3 cells.

and C). Noticeably, the mTOR signaling pathway, one of the common activated pathways in $\mathrm{HCC}^{21}$ was significantly downregulated in the plumbagin-treated group (Figure 4C) and the $-\log 10(P$-value) was 6.99 . Western blotting results confirmed that SIVA was inhibited by plumbagin in a dose-dependent manner in both HepG2 and LM3 cells (Figure 5A). SIVA was reported to be a p53 targeted gene, however, no significant difference was found regarding the expression of p53 (Figure 5A). Meanwhile, we detected the
mTOR signaling, and results revealed that mTOR signaling was inhibited in plumbagin-treat cells in a dosedependent manner (Figure 5A). Then we downregulated the expression of SIVA to clarify its role in regulating the mTOR pathway. Results showed that the downstream molecules of the mTOR pathway, $\mathrm{p}-\mathrm{S} 6 \mathrm{~K}$, and p-4EBP1 were suppressed in the SIVAknockdown group (Figure 5B), which further demonstrated that PL inhibited the mTOR pathway by downregulating the expression of SIVA. 
A
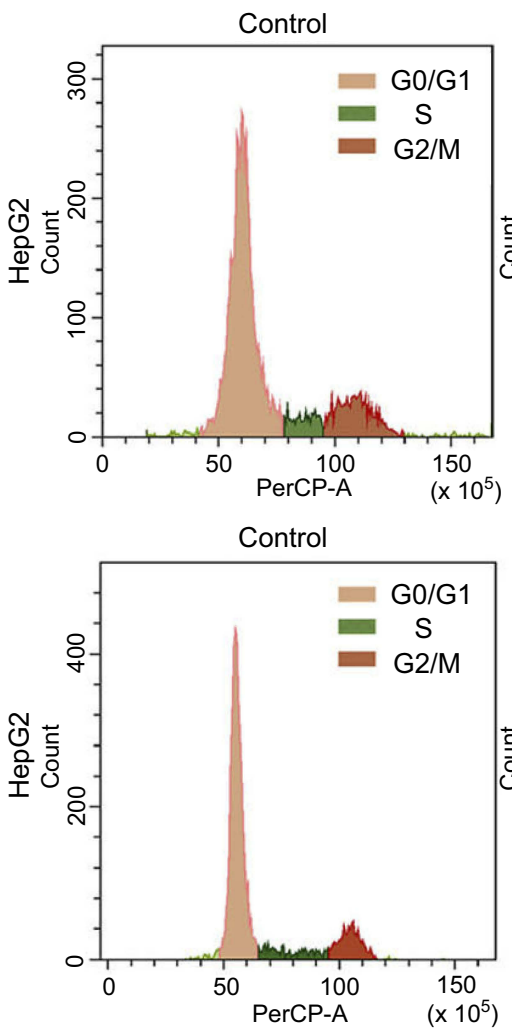
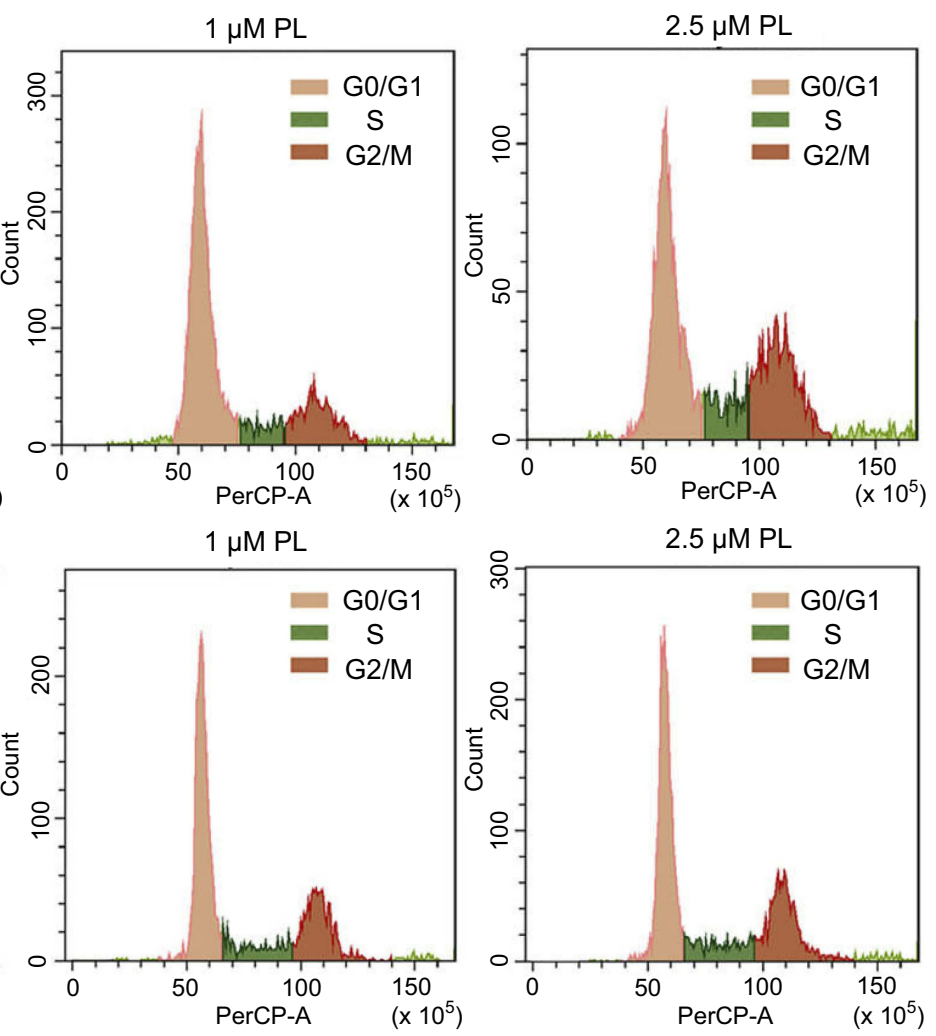

B
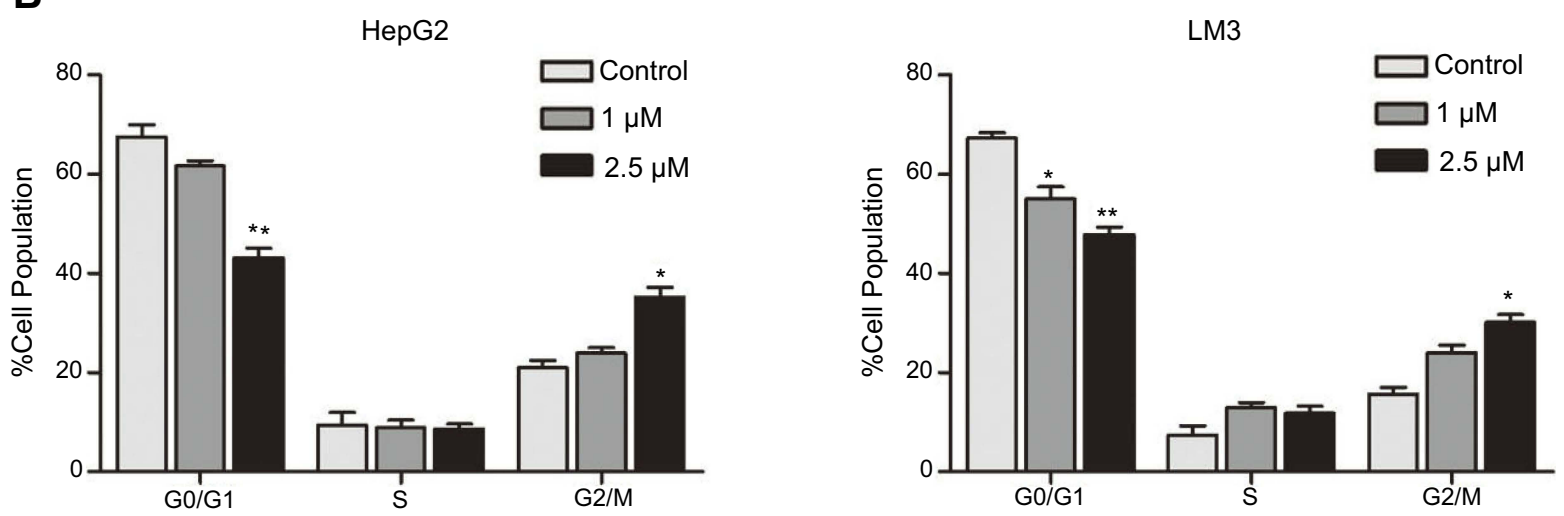

Figure 2 Plumbagin arrests the progression of the cell cycle in HepG2 and LM3 cells. (A) The cell cycle distribution detected by flow cytometry. (B) Statistical results of cell cycle detection; $* P<0.05, * * P<0.0$ I vs control group.

\section{SIVA plays a pivotal role in inducing apoptosis of HCC by plumbagin}

We overexpressed SIVA in HCC cells to clarify SIVA's role in inhibiting plumbagin-treated cells (Figure 6). To be specific, the inhibitory effect of plumbagin on the mTOR signaling pathway was alleviated by overexpressing SIVA (Figure 6A). Activating the mTOR signaling pathway can lighten the antiproliferative activity of plumbagin on $\mathrm{HCC}$ cells (Figure $6 \mathrm{~B})$. In addition, the effects of plumbagin on colony forming capacity and apoptosis rate were also rescued by overexpressing SIVA (Figures 6C and D). These results suggested that PL's cytotoxicity against HCC was mediated by SIVA, which could attenuate the mTOR pathway.

\section{Plumbagin suppresses the tumorigenicity of $\mathrm{HCC}$}

A xenograft model was built by injecting luciferase-marked HCC cells in the nude mouse. The intraperitoneal administration of plumbagin can significantly inhibit the growth of tumor (Figure 7A). Furthermore, the plumbagin-treated group 
A
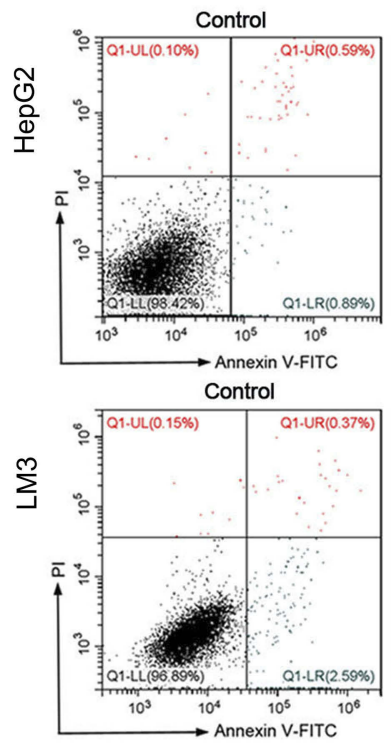
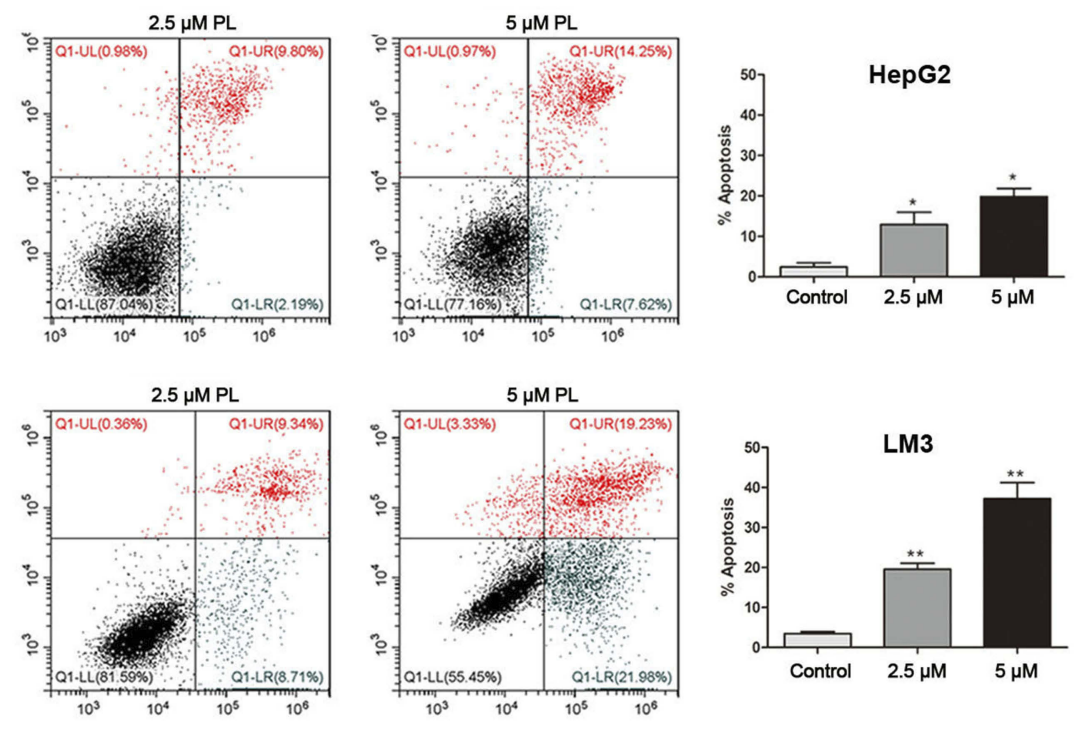

B

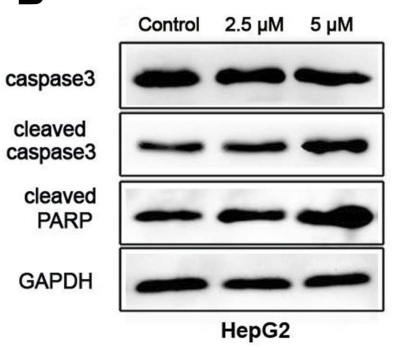

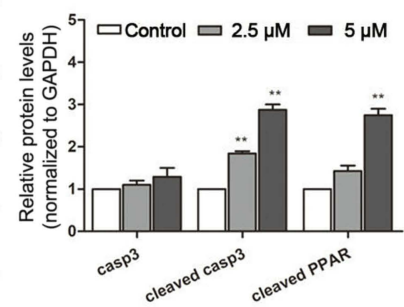

Figure 3 Plumbagin induces apoptosis in HepG2 and LM3 cells. (A) Results of cell apoptosis examined by Annexin-V/PI staining using flow cytometry. *P<0.05, $* * P<0.01$ vs control group. (B) The expression of caspase and PARP detected by Western blotting. $* P<0.05, * * P<0.01$ vs control group.

exhibited a smaller tumor volume and longer survival period than the control group (Figure 7B). Immunohistochemistry of the tumor tissue showed that the SIVA and p-mTOR were downregulated in the plumbagin-treated group (Figure 7C), which was well in line with our in vitro results that plumbagin owned antitumor efficacy against HCC through inhibiting the SIVA/mTOR signaling pathway.

\section{Discussion}

Hepatocellular carcinoma (HCC) is one of the most common solid cancer in China, which is characterized with a high metastatic rate and poor prognosis. ${ }^{22}$ Despite some progress made in the treatment on $\mathrm{HCC}$, the overall 5 -year survival rate is still lower than $40 \%{ }^{22}$ Specifically, some patients were diagnosed with $\mathrm{HCC}$ at an advanced stage and missed the opportunity for hepatectomy. Meanwhile, the chemotherapy resistance of HCC also contributes to the poor prognosis. ${ }^{23}$ Herein, we introduce a traditional herbal medicine, plumbagin, to serve as an anti-tumor agent against HCC. Plumbagin is one of the extracts of plumbago indica L. Some other extracts of plumbago indica $L$ also show some specific biological effects. For example, $\beta$-sitosterol and $\beta$ trilinolein, two extracts of plumbago indica $\mathrm{L}^{24}$, exhibited cytotoxicity against breast cancer cell MCF7 and had an anti-inflammatory effect, respectively. ${ }^{24,25}$ Plumbagin was also reported to show antibacterial activity against Staphylococcus aureus and Staphylococcus epidermidis. ${ }^{26}$ Recently, some studies have shown that plumbagin exerted an anti-tumor effect on some tumors, including prostate cancer, esophageal cancer, and lung cancer. ${ }^{14,15,17}$ To be specific, plumbagin can inhibit proliferation and induce apoptosis of esophageal cancer cells through downregulating the STAT3-PLK1-AKT signaling pathway. ${ }^{14}$ Regarding non-small cell lung cancer, plumbagin enhanced autophagy and apoptosis of non-small cell lung cancer cells by inhibition of the PI3K/Akt/ mTOR pathway. ${ }^{17}$ Meanwhile, plumbagin also acted as an inhibitor of the growth and invasion of prostate cancer through inhibiting protein kinase C. ${ }^{27}$ However, few 

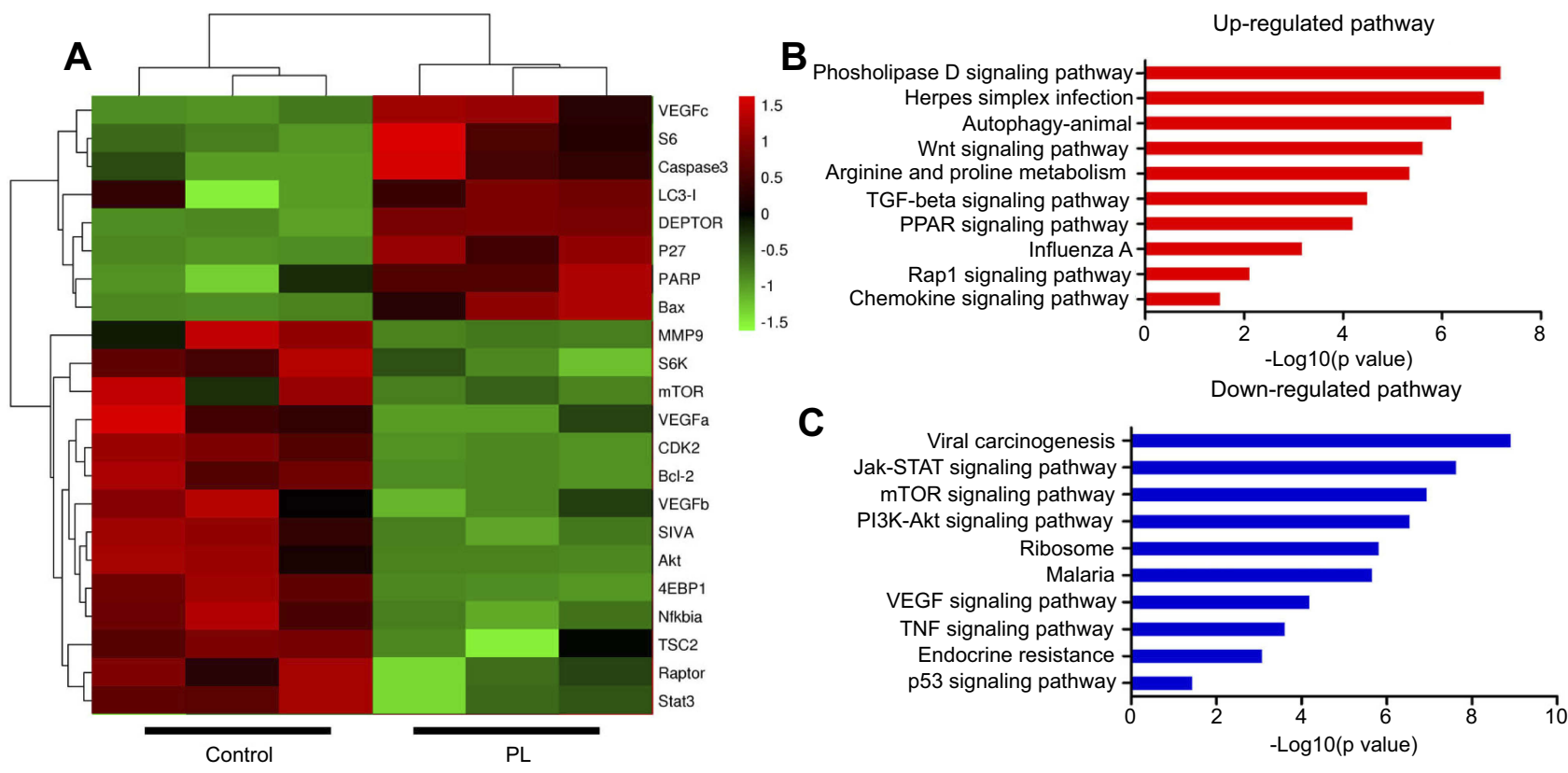

Figure 4 The gene expression profile of plumbagin-treated cells detected by RNA-seq. (A) Heat map depicting the fold change of representative genes in plumbagin-treated LM3 cells. (B and $\mathbf{C}$ ) Top 10 upregulated or downregulated signaling pathway analyzed by KEGG in plumbagin-treated LM3 cells.

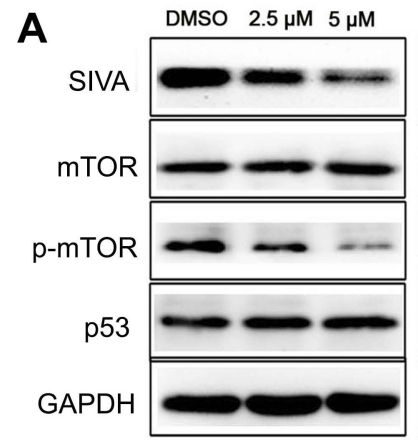

HepG2

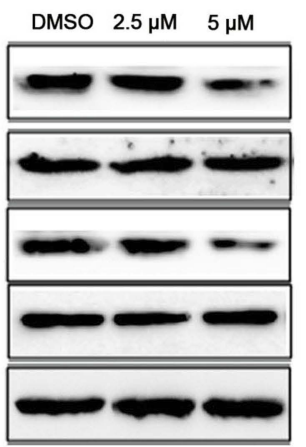

LM3
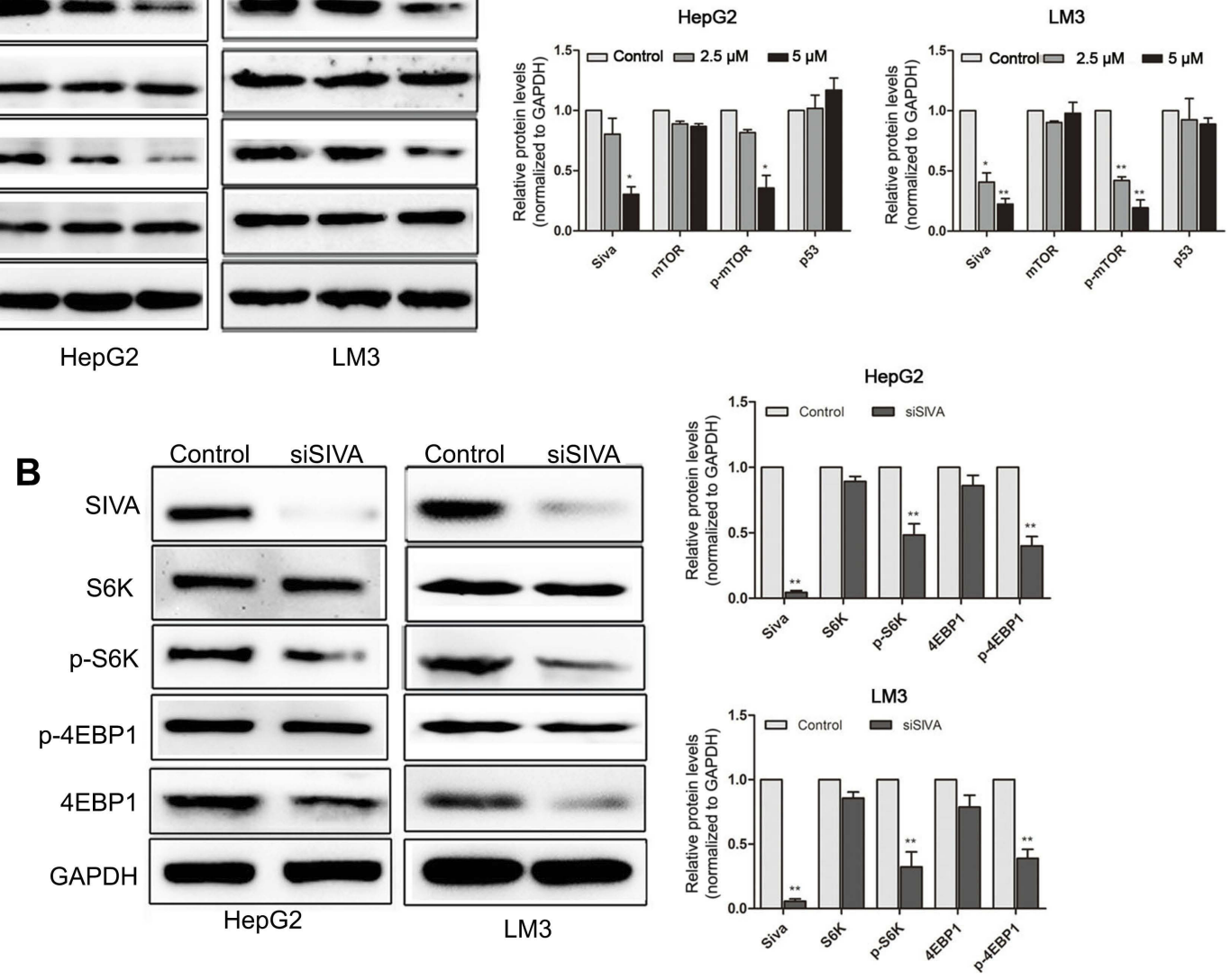

Figure 5 (A) The Western blotting results of SIVA, mTOR, and $p 53$ when treated with $2.5 \mu \mathrm{M}, 5 \mu \mathrm{M}$ plumbagin for 48 hours in HepG2 and LM3 cells. $* P<0.05$, $* * P<0.01$ vs control group. (B) The expression of mTOR downstream molecules (S6K and 4EBPI) in SIVA-knockdown HepG2 and LM3 cells. **P<0.0I vs control group. 


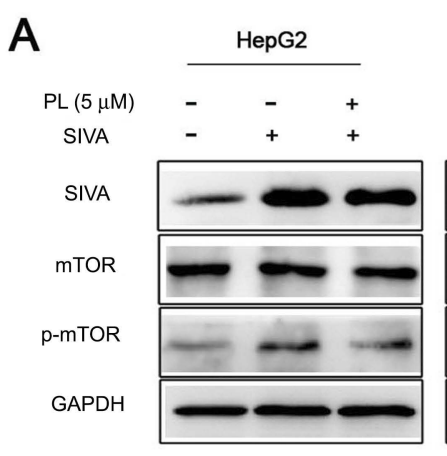

B

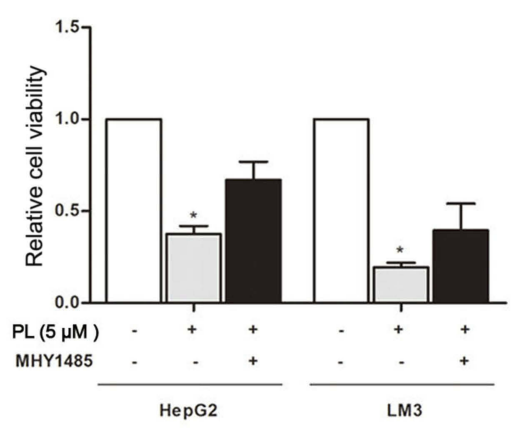

D
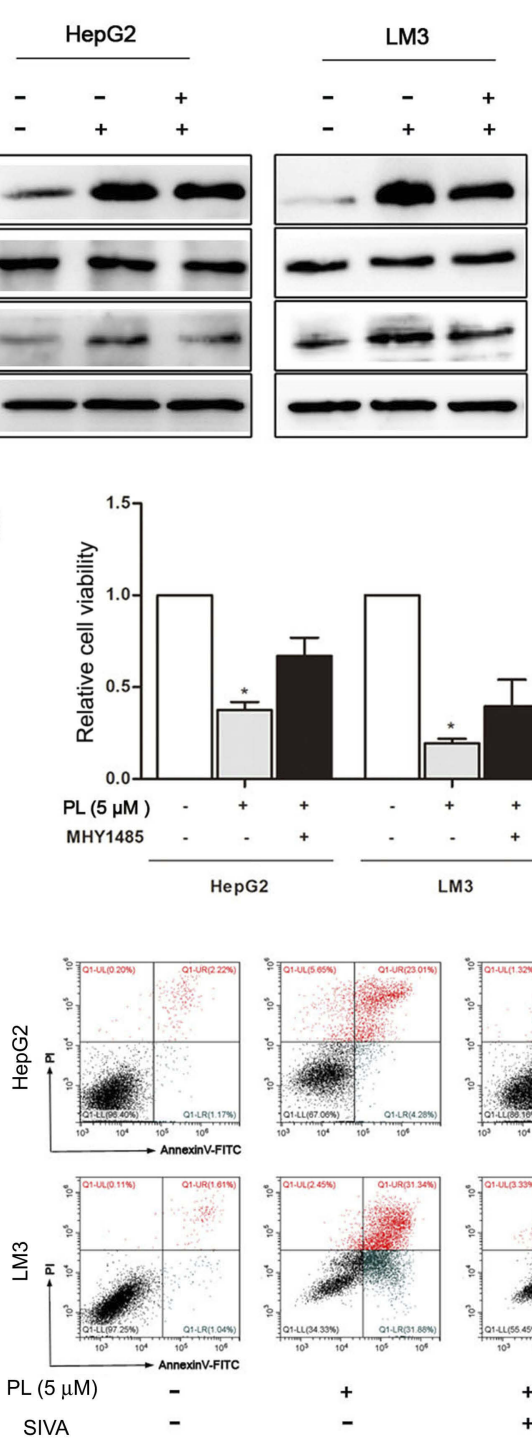
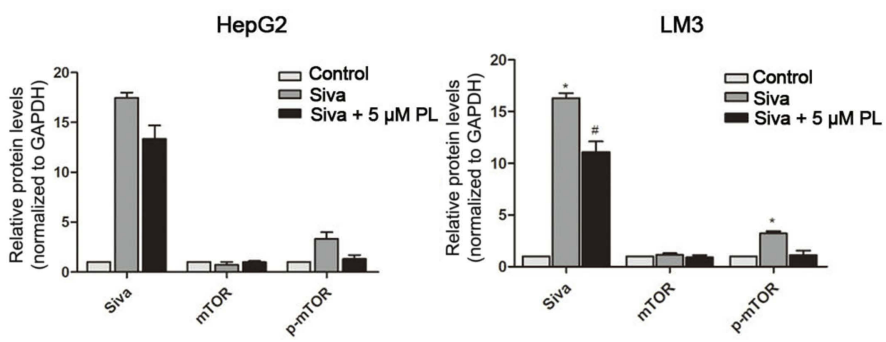

C
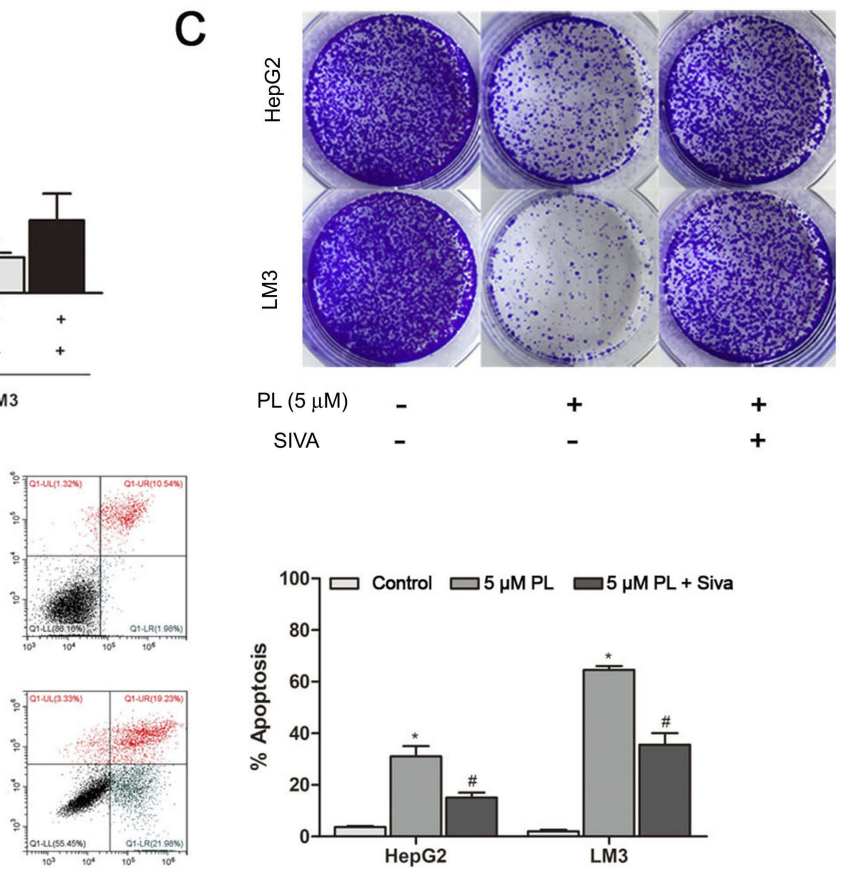

Figure 6 Plumbagin inhibits proliferation of HCC cells through inhibiting the IVA/mTOR pathway. (A) The expression of SIVA and mTOR in SIVA-overexpressed HepG2 and LM3 cells when treated with plumbagin. ${ }^{*} P<0.05$ vs control group, ${ }^{\#} P<0.05$ vs Siva overexpressed group. (B) Cell viability of plumbagin-treated cells when activating the mTOR signal pathway. ${ }^{*} P<0.05$ vs control group. (C) Representative images of colony forming test in SIVA-overexpressed cells when treated with plumbagin. (D) Results of cell apoptosis examined by Annexin-V/PI staining in SIVA-overexpressed cells when treated with plumbagin. ${ }^{* P}<0.05$ vs control group, ${ }^{\#} P<0.05$ vs $\mathrm{PL}$ group.

studies have focused on the anti-tumor activity of plumbagin on HCC. Our current study demonstrates the antitumor activity of plumbagin against HCC. Two types of HCC cells (HepG2 and Lm3) were employed. The CCK8, Edu, and CFE tests revealed that plumbagin showed antiproliferative activity against $\mathrm{HCC}$ cells, especially LM3 cells. Cell cycle and apoptosis analyses were done through a flow cytometer and Western blotting. Results turn out that plumbagin can induce cell cycle arrest at $\mathrm{G} 2 / \mathrm{M}$ phase and decrease the proportion of $\mathrm{G} 0 / \mathrm{G} 1$ phase. Besides, the apoptotic population was enhanced in the plumbagin-treated group, which was consistent with results of Western blotting.
However, leaving alone the molecular mechanism of cancer proliferation and invasion will never lead to an ideal treatment or drug against cancer cells. It is well known that several signaling pathways are indispensable in tumorigenesis and progression in $\mathrm{HCC}$, including the Wnt/beta-catenin signaling pathway, Notch signaling pathway, and mTOR signaling pathway. ${ }^{28-30}$ To further investigate the molecular mechanism that plumbagin inhibits proliferation of HCC cells, we first performed an RNA seq to acquire the expression profile of plumbagin-treated cells. Among the fold changes genes, a p53 targeted gene SIVA has drawn our attention. SIVA, a proapoptotic protein, was originally identified 
A
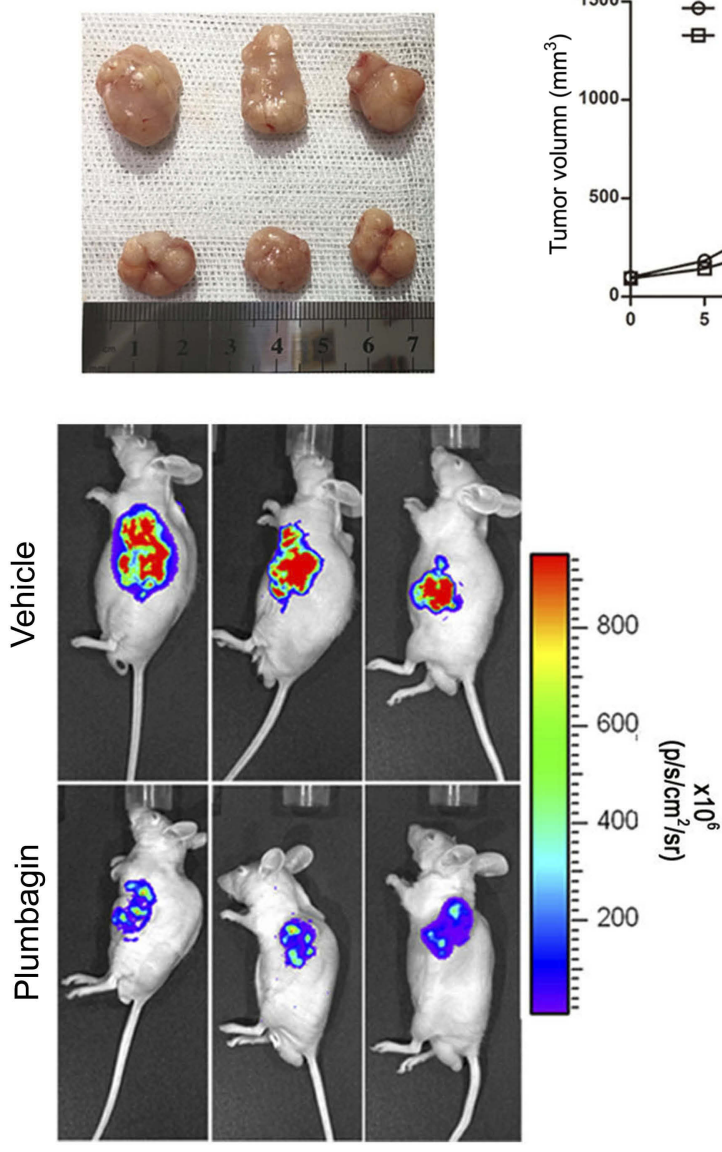

B
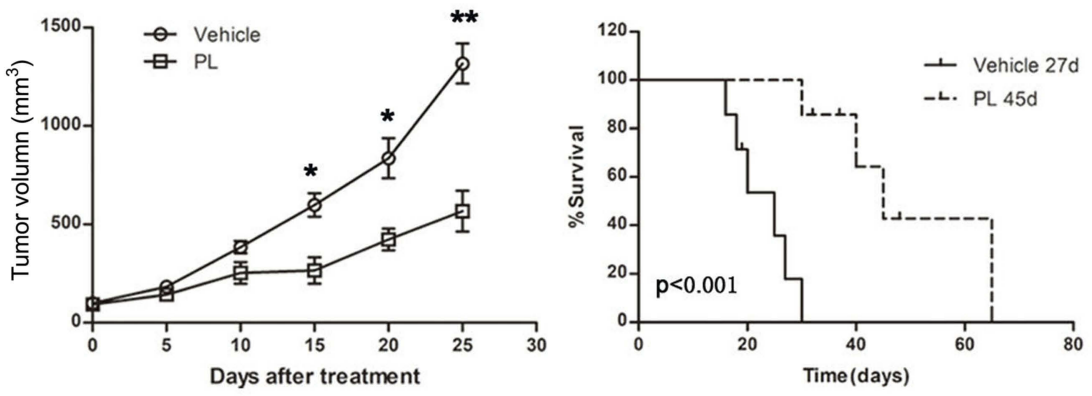

C
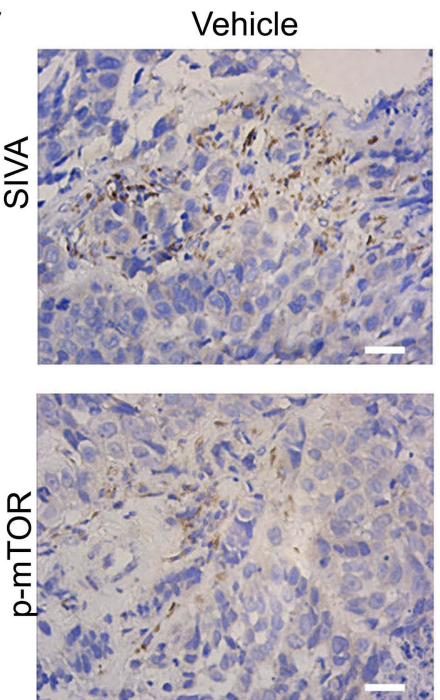

Plumbagin
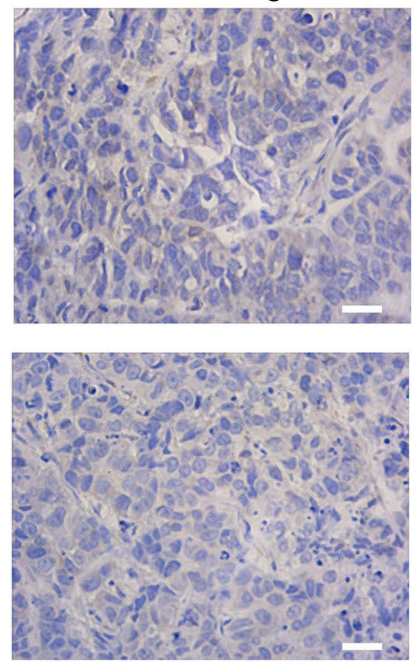

Figure 7 Plumbagin suppresses in vivo tumorigenicity. (A) Representative images of tumor gross appearance (upper) and bioluminescence (lower). (B) The statistical results of tumor volume (left) and animal survival period (right). $* P<0.05$ and $* * P<0.01$ vs control group. (C) Representative images of immunohistochemistry depicting the expression of SIVA and p-mTOR, bar: $50 \mu \mathrm{m}$.

through its interaction with some death receptors, ${ }^{31}$ for example CD27. ${ }^{19}$ It was reported that SIVA participated in p53-dependent apoptosis and was an important regulator of the interaction between $\mathrm{p} 53$ and $\mathrm{Hdm} 2 .^{32}$ Moreover, recent studies demonstrated that inhibiting expression of SIVA can exert tumor-suppressive and anti-metastatic effects on kinds of cancers. $^{20,33}$ Meanwhile, studies have revealed that SIVA can serve as a p53 targeted gene or p53-independent gene (a mTOR signaling activator) to realize its functions. ${ }^{20,34}$ To figure out the exact role that SIVA plays in the plumbagin-treated cells, we performed Western blotting to determine the expression of SIVA, p53, and mTOR in plumbagin-treated cells. As shown in Figure 5A, SIVA was downregulated when treated with plumbagin following a concentration and time dependent manner.
Meanwhile, the inhibition of mTOR was observed, but no significant difference was found about the expression of p53 in the plumbagin-treated group, which was consistent with a former study that SIVA can act independently of p53 and stimulate the mTOR signaling pathway. We further found that the expression of mTOR was inhibited when SIVA was knocked down in HCC cells. Therefore, we argued that plumbagin can inhibit the mTOR signaling pathway through downregulating SIVA but not p53.

It is well known that the mammalian target of rapamycin (mTOR) kinase, a conserved serine/threonine protein kinase, plays a crucial role in regulating cell cycle and cell growth. ${ }^{35,36}$ Moreover, the mTOR signaling pathway is proven to modulate some cellular processes, including protein synthesis, cell growth and 
proliferation, cell death, and tumor angiogenesis. ${ }^{37,38}$ The hyperactivation of the mTOR signaling pathway can be observed in many malignant tumors, and the mTOR signaling pathway is considered to be a potential target for anti-tumor therapy. ${ }^{39,40}$ In the present study, the inhibition of the mTOR signaling pathway, SIVA, and cell proliferation, apoptosis was observed when treated with plumbagin in HCC cells. To investigate whether the inhibition of SIVA, mTOR signaling pathway results in an anti-tumor effect in the plumbagin-treated group, we upregulated the expression of SIVA or activated the mTOR signaling pathway through adding an activator to plumbagin-treated cells and then evaluated cell viability and apoptosis. Our data demonstrated the anti-tumor activity of plumbagin against HCC cells was significantly reversed by overexpressing SIVA or activating the mTOR signaling pathway. The in vivo anti-tumor activity of plumbagin was evaluated by xenograft model. The results of in vivo experiments were in line with in vitro studies that plumbagin can inhibit HCC proliferation through inhibiting SIVA and the mTOR signaling pathway.

\section{Conclusion}

In a word, our current study has demonstrated the antitumor activity of plumbagin against $\mathrm{HCC}$, which may attribute to plumbagin's inhibition of the SIVA/mTOR signaling pathway. Our promising results indicate that plumbagin is a potential therapeutic agent against HCC, and this study may pave the way for plumbagin's further application as a good candidate for overcoming drug resistance about HCC.

\section{Abbreviation list}

HCC, hepatocellular carcinoma; PBS, phosphate buffered saline; DMEM, Dulbecco's modified eagle medium; CCK8, cell counting kit-8; EdU, 5-ethynyl-2'-deoxyuridin; PI, propidiumiodide; PFA, paraformaldehyde; RNA-seq,pribose) polymerase.

\section{Acknowledgment}

This work was supported by the National Natural Science Foundation of China (81470853).

\section{Disclosure}

The authors report no conflicts of interest in this work.

\section{References}

1. El-Serag HB. Hepatocellular carcinoma. New England $J$ Med. 2011;365(12):1118-1127. doi:10.1056/NEJMra1001683

2. Ferlay J, Soerjomataram I, Dikshit R, et al. Cancer incidence and mortality worldwide: sources, methods and major patterns in GLOBOCAN 2012. Int $J$ Cancer. 2015;136(5):E359-E386. doi:10.1002/ijc. 29210

3. Skolnick AA. Armed with epidemiologic research, China launches programs to prevent liver cancer. Jama. 1996;276(18):1458-1459.

4. Wallace MC, Preen D, Jeffrey GP, Adams LA. The evolving epidemiology of hepatocellular carcinoma: a global perspective. Expert Rev Gastroenterol Hepatol. 2015;9(6):765-779. doi:10.1586/ 17474124.2015.1028363

5. Attwa MH, El-Etreby SA. Guide for diagnosis and treatment of hepatocellular carcinoma. World J Hepatol. 2015;7(12):1632-1651. doi: $10.4254 /$ wjh.v7.i12.1632

6. Mokdad AA, Hester CA, Singal AG, Yopp AC. Management of hepatocellular in the United States. Chin Clin Oncol. 2017;6(2):21. doi: $10.21037 / \mathrm{cco}$

7. Galle PR, Tovoli F, Foerster F, Worns MA, Cucchetti A, Bolondi L. The treatment of intermediate stage tumours beyond TACE: from surgery to systemic therapy. $J$ Hepatol. 2017;67(1):173-183. doi:10.1016/j.jhep.2017.03.007

8. Bruix J, Reig M, Sherman M. Evidence-based diagnosis, staging, and treatment of patients with hepatocellular carcinoma. Gastroenterol. 2016;150(4):835-853. doi:10.1053/j.gastro.2015.12.041

9. Bruix J, Gores GJ, Mazzaferro V. Hepatocellular carcinoma: clinical frontiers and perspectives. Gut. 2014;63(5):844-855. doi:10.1136/ gutjnl-2013-306627

10. Zhang YS, Ma YL, Thakur K, et al. Molecular mechanism and inhibitory targets of dioscin in HepG2 cells. Food Chem Toxicol. 2018;120:143-154. doi:10.1016/j.fct.2018.07.016

11. Wang J, Zhang YS, Thakur K, et al. Licochalcone A from licorice root, an inhibitor of human hepatoma cell growth via induction of cell apoptosis and cell cycle arrest. Food Chem Toxicol. 2018;120:407-417. doi:10.1016/j.fct.2018.07.044

12. Liu Y, Cai Y, He C, Chen M, Li H. Anticancer properties and pharmaceutical applications of plumbagin: a review. Am $J$ Chin Med. 2017;45(3):423-441. doi:10.1142/S0192415X17500264

13. Panichayupakaranant $P, A$ hmad MI. Plumbagin and its role in chronic diseases. Adv Exp Med Biol. 2016;929:229-246. doi:10.1007/9783-319-41342-6_10

14. Cao YY, Yu J, Liu TT, et al. Plumbagin inhibits the proliferation and survival of esophageal cancer cells by blocking STAT3-PLK1-AKT signaling. Cell Death Dis. 2018;9(2):17. doi:10.1038/s41419-017-0068-6

15. Hafeez BB, Zhong W, Mustafa A, Fischer JW, Witkowsky O, Verma AK. Plumbagin inhibits prostate cancer development in TRAMP mice via targeting PKCepsilon, Stat3 and neuroendocrine markers. Carcinog. 2012;33(12):2586-2592. doi:10.1093/carcin/bgs291

16. Sugie S, Okamoto K, Rahman KM, et al. Inhibitory effects of plumbagin and juglone on azoxymethane-induced intestinal carcinogenesis in rats. Cancer Letters. 1998;127(1-2):177-183.

17. $\mathrm{Li} \mathrm{YC}, \mathrm{He} \mathrm{SM}, \mathrm{He} \mathrm{ZX}$, et al. Plumbagin induces apoptotic and autophagic cell death through inhibition of the PI3K/Akt/mTOR pathway in human non-small cell lung cancer cells. Cancer Letters. 2014;344(2):239-259. doi:10.1016/j.canlet.2013.11.001

18. Hafeez BB, Jamal MS, Fischer JW, Mustafa A, Verma AK. Plumbagin, a plant derived natural agent inhibits the growth of pancreatic cancer cells in in vitro and in vivo via targeting EGFR, Stat3 and NF- $\mathrm{KB}$ signaling pathways. Int $J$ Cancer. 2012;131 (9):2175-2186. doi:10.1002/ijc. 27478

19. Prasad KV, Ao Z, Yoon Y, et al. CD27, a member of the tumor necrosis factor receptor family, induces apoptosis and binds to Siva, a proapoptotic protein. Pro National Acad Sci USA. 1997;94 (12):6346-6351 
20. Van Nostrand JL, Brisac A, Mello SS, Jacobs SB, Luong R, Attardi LD. The p53 target gene SIVA enables non-small cell lung cancer development. Cancer Discovery. 2015;5(6):622-635. doi:10.1158/2159-8290.CD-14-0921

21. Matter MS, Decaens T, Andersen JB, Thorgeirsson SS. Targeting the mTOR pathway in hepatocellular carcinoma: current state and future trends. $J$ Hepatol. 2014;60(4):855-865. doi:10.1016/j. jhep.2013.11.031

22. Chen W, Zheng R, Baade PD, et al. Cancer statistics in China, 2015. CA. 2016;66(2):115-132. doi:10.3322/caac.21338

23. Ray EM, Sanoff HK. Optimal therapy for patients with hepatocellular carcinoma and resistance or intolerance to sorafenib: challenges and solutions. J Hepatocellular Carcinoma. 2017;4:131-138. doi:10.2147/JHC.S124366

24. Nguyen AT, Malonne H, Duez P, Vanhaelen-Fastre R, Vanhaelen M, Fontaine J. Cytotoxic constituents from Plumbago zeylanica. Fitoterapia. 2004;75(5):500-504. doi:10.1016/j.fitote.2004.03.009

25. Huang SS, Deng JS, Lin JG, Lee CY, Huang GJ. Anti-inflammatory effects of trilinolein from Panax notoginseng through the suppression of NF-kappaB and MAPK expression and proinflammatory cytokine expression. Am J Chin Med. 2014;42(6):1485-1506. doi:10.1142/ S0192415X14500931

26. Kaewbumrung S, Panichayupakaranant P. Antibacterial activity of plumbagin derivative-rich Plumbago indica root extracts and chemical stability. Nat Product Res. 2014;28(11):835-837. doi:10.1080/ 14786419.2013.879585

27. Aziz MH, Dreckschmidt NE, Verma AK. Plumbagin, a medicinal plant-derived naphthoquinone, is a novel inhibitor of the growth and invasion of hormone-refractory prostate cancer. Cancer Res. 2008;68 (21):9024-9032. doi:10.1158/0008-5472.CAN-08-2494

28. Zhan T, Rindtorff N, Boutros M. Wnt signaling in cancer. Oncogene. 2017;36(11):1461-1473. doi:10.1038/onc.2016.304

29. Li L, Tang P, Li S, et al. Notch signaling pathway networks in cancer metastasis: a new target for cancer therapy. Med Oncol. 2017;34 (10):180. doi:10.1007/s12032-017-1039-6
30. Martini M, De Santis MC, Braccini L, Gulluni F, Hirsch E. PI3K/ AKT signaling pathway and cancer: an updated review. Ann Med. 2014;46(6):372-383. doi:10.3109/07853890.2014.912836

31. Jacobs SB, Basak S, Murray JI, Pathak N, Attardi LD. Siva is an apoptosis-selective p53 target gene important for neuronal cell death. Cell Death Differ. 2007;14(7):1374-1385. doi:10.1038/sj.cdd.4402128

32. Higashitsuji $\mathrm{H}$, Higashitsuji $\mathrm{H}$, Itoh $\mathrm{K}$, et al. The oncoprotein gankyrin binds to MDM2/HDM2, enhancing ubiquitylation and degradation of p53. Cancer Cell. 2005;8(1):75-87. doi:10.1016/j. ccr.2005.06.006

33. Li N, Jiang P, Du W, et al. Sival suppresses epithelial-mesenchymal transition and metastasis of tumor cells by inhibiting stathmin and stabilizing microtubules. Pro National Acad Sci USA. 2011;108 (31):12851-12856. doi:10.1073/pnas.1017372108

34. Fortin A, MacLaurin JG, Arbour N, et al. The proapoptotic gene SIVA is a direct transcriptional target for the tumor suppressors p53 and E2F1. J Biol Chem. 2004;279(27):28706-28714. doi:10.1074/ jbc.M400376200

35. Sabatini DM, Erdjument-Bromage H, Lui M, Tempst P, Snyder SH. RAFT1: a mammalian protein that binds to FKBP12 in a rapamycin-dependent fashion and is homologous to yeast TORs. Cell. 1994;78(1):35-43.

36. Heitman J, Movva NR, Hall MN. Targets for cell cycle arrest by the immunosuppressant rapamycin in yeast. Science. 1991;253 (5022):905-909.

37. Wullschleger S, Loewith R, Hall MN. TOR signaling in growth and metabolism. Cell. 2006;124(3):471-484. doi:10.1016/j.cell.2006.01.016

38. Guertin DA, Sabatini DM. An expanding role for mTOR in cancer. Trends in Molecular Medicine. 2005;11(8):353-361. doi:10.1016/j. molmed.2005.06.007

39. Populo H, Lopes JM, Soares P. The mTOR signalling pathway in human cancer. Int J Mol Sci. 2012;13(2):1886-1918. doi:10.3390/ ijms 13021886

40. Easton JB, Houghton PJ. mTOR and cancer therapy. Oncogene. 2006;25(48):6436-6446. doi:10.1038/sj.onc.1209886
Drug Design, Development and Therapy

\section{Publish your work in this journal}

Drug Design, Development and Therapy is an international, peerreviewed open-access journal that spans the spectrum of drug design and development through to clinical applications. Clinical outcomes, patient safety, and programs for the development and effective, safe, and sustained use of medicines are a feature of the journal, which has also been accepted for indexing on PubMed Central. The manuscript management system is completely online and includes a very quick and fair peer-review system, which is all easy to use. Visit http://www. dovepress.com/testimonials.php to read real quotes from published authors. 